\title{
Morphological characterization of the nasopalatine region in human fetuses and its association to pathologies
}

\author{
Saulo Gabriel Moreira FALCI' Flaviana Dornela VERLI $^{2}$, Alberto CONSOLARO ${ }^{3}$, Cássio Roberto Rocha dos SANTOS ${ }^{4}$
}

1- DDS, MSc in Dental Clinic, School of Dentistry, Federal University of Vales do Jequitinhonha e Mucuri, Diamantina, MG, Brazil.

2- DDS, MSc, PhD in Stomatology, Department of Basic Sciences, Federal University of Vales do Jequitinhonha e Mucuri, Diamantina, MG, Brazil.

3- DDS, MSc, PhD in Oral Pathology, Department of Stomatology, Bauru School of Dentistry, University of São Paulo, Bauru, SP, Brazil.

4- DDS, MSc, PhD in Oral Pathology, Department of Oral and Maxillofacial Surgery, Federal University of Vales do Jequitinhonha e Mucuri, Diamantina, MG, Brazil.

Corresponding address: Saulo Gabriel Moreira Falci, - Rua Tiradentes, 195E - Diamantina - MG - Brasil - $39100-000$ - Phone/fax: +55 38 3532-6000 e-mail: saulofalci@hotmail.com

Received: January 7, 2013 - Modification: March 5, 2013 - Accepted: May 2, 2013

\section{ABSTRACT}

$\mathrm{T}$ he nasopalatine region is composed of structures such as the vomeronasal organ and nasopalatine duct. The nasopalatine duct may provide the communication of the mouth to the nasal cavity in human fetuses and can be obliterated in an adult human. Knowledge on the development of the nasopalatine region and nasopalatine duct in humans is necessary for understanding the morphology and etiopathogenesis of lesions that occur in this region. Objective: The aim of the present study was to describe the morphological aspects of the nasopalatine region in human fetuses and correlate these aspects with the development of pathologies in this region. Material and Methods: Five human fetuses with no facial or palatine abnormalities were used for the acquisition of specimens from the nasopalatine region. After demineralization, the specimens were histologically processed. Histological cuts were stained with methylene blue to orient the cutting plane and hematoxylin-eosin for the descriptive histological analysis. Results: The age of the fetuses was 8.00, 8.25, 9.00 and 9.25 weeks, and it was not possible to determine the age in the last one. The incisive canal was observed in all specimens as an opening delimited laterally by the periosteum and connecting oral and nasal cavity. The nasopalatine duct is an epithelial structure with the greatest morphological variation, with either unilateral or bilateral occurrence and total patent, partial patent and islet forms. The vomeronasal organ is a bilateral epithelized structure located alongside the nasal septum above the incisive canal in all the fetuses. Conclusions: The incisive canal, nasopalatine duct and vomeronasal organ are distinct anatomic structures. The development of nasopalatine duct cysts may occur in all forms of the nasopalatine duct.

Key words: Morphogenesis. Maxilla. Vomeronasal organ. Palate. Incisive papilla.

\section{INTRODUCTION}

In most mammals, the nasopalatine duct is responsible for the conduction of pheromones from the mouth to the nasal cavity, thereby assisting in communication and breeding ${ }^{18}$. The duct generally terminates in the floor of the nasal cavity near the vomeronasal organ. This organ is part of the accessory olfactory system in mammals and is responsible for the capturing of pheromones and neural communication with the central nervous system $^{18,19}$.

The function of the vomeronasal organ in humans is contested, since the nasopalatine duct can become blocked following birth7,15,16. At times, the nasopalatine duct can remain patent, maintaining communication between the oral cavity and nasal cavity. The vestigial form of the nasopalatine duct in humans may cause problems, such as the development of a nasopalatine duct cyst, which is the most frequent non-odontogenic cyst in the maxilla ${ }^{9,13,19,21,23}$. The development, 
function and persistence of the vomeronasal organ and nasopalatine duct in animals has been the topic of studies ${ }^{18-20}$. Knowledge on the development of the nasopalatine region and nasopalatine duct in humans is necessary for understanding the morphology of this region and the establishment of theories that may clarify the etiopathogenesis of lesions that occur in this anatomic region. A recent published paper regarding histopathogenesis of a nasopalatine duct cyst suggests that this lesion can be an inflammatory cyst, like a radicular cyst or a salivary duct cyst-like mucocele ${ }^{22}$. However, to our understanding, the etiophatogenesis of this lesion remains unclear due to the reduced number of articles published about it.

The aims of the present study were to analyze the morphological aspects of the nasopalatine region in human fetuses to determine the presence of the nasopalatine duct and vomeronasal organ and describe the morphology of the incisive canal and characteristics of the mucosa and submucosa, and correlating these characteristics with the development of pathologies in the anterior region of the maxilla.

\section{MATERIAL AND METHODS}

\section{Sample}

Five non identified fetuses were obtained from the Anatomic Bank of the Oral Pathology Sector of the authors' University. The fetuses were submerged in a $10 \%$ formalin solution (Synth, Diadema, Brazil). The inclusion criterion for the selection of the fetuses was the absence of facial and palatine abnormalities, as observed macroscopically. The age of the fetuses was determined based on femur size $^{11}$.

\section{Macroscopic analysis}

The five fetuses were decapitated and the heads were frozen at a temperature of $-22^{\circ} \mathrm{C}$ for 24 hours. Two sagittal cuts tangent to the wing of the nose were then performed on the right and left sides with the aid of a circular electric saw (Bosch, São Paulo, Brazil). On the sagittal cut, three other cuts were performed - one coronal and two axial. The coronal cut passed through the pharynx and posterior region of the soft palate. One axial cut began at the tip of the nose parallel to the base of the skull base and the other at the lip commissures and oral cavity. These axial cuts were performed from their points of origin to the coronal cut (Figure 1A). The resulting piece was made up of the anterior region of the hard palate and the nasal cavity. This piece was submitted to radiographic analysis with E041 occlusal, intra-oral film (Kodak, Rochester, USA) for detection of the trajectory of the incisive canal (Figure 1B).

The rectangular specimen was divided into three segments obtained through two incisions. The first incision was made in the vestibular fornix and the second was made between the soft and hard palate. Thus, the first segment was comprised of the upper lip and nose tip; the second segment involved the hard palate; and the third segment was made up of the oropharynx and soft palate. Due to the presence of bone tissue, the second segment was demineralized in $12.5 \%$ ethylenediaminetetraacetic acid (Dinâmica Química Contemporânea, São Paulo, Brazil) for six weeks in a glass recipient. The rest of the head was kept in a recipient containing a $10 \%$ formalin solution.

\section{Histological processing}

The second segment was submitted to routine histological procedures and embedded in a single
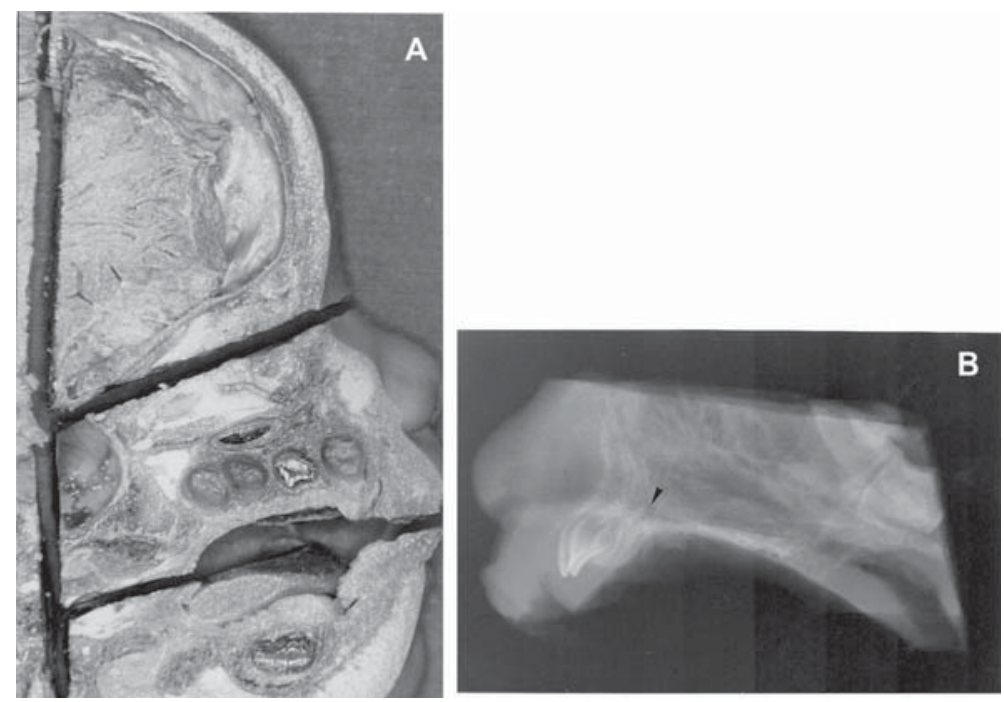

Figure 1- Cuts performed on head of fetus for histological processing in A. Lateral radiograph of final piece revealing trajectory of incisive canal in B (arrow) 
paraffin block. The segment was positioned for longitudinal histological cuts parallel to the trajectory of the incisive canal (located through radiographs). Cuts with $6-\mu \mathrm{m}$ thicknesses were performed and mounted on glass slides for microscopic analysis. The pairs of slides were stained with methylene blue (Synth, Diadema, Brazil) and used to orient the preestablished cutting plane. The cuts were performed up to the end of the incisive canal. From the pairs of slides, only the odd slides were stained with hematoxylin-eosin (HE) (Synth, Diadema, Brazil) and cover slips were placed over the specimens.

\section{Microscopic analysis}

The slides were examined under a light microscope (Carl Zeiss, Gottingen, Germany). The following histological variables were analyzed: (1) shape and histological aspects of the incisive canal; (2) presence, shape and histological aspects of the nasopalatine duct; (3) location and histological aspects of the vomeronasal organ; and (4) histological aspects of the mucosa, submucosa and ossification of the nasopalatine region.

The study received approval from the ethics committee of the author's University.

\section{RESULTS}

\section{Age and gender of fetuses}

The age of the fetuses was $8.00,8.25,9.00$ and 9.25 weeks. Three fetuses were male and one was female. It was not possible to determine the age and gender of one fetus because only the head was available.

\section{Microscopic analysis}

The nasopalatine region exhibited similar histological aspects among the five fetuses. On all longitudinal histological cuts, the nasopalatine region was delimited by the oral and nasal cavities. The oral cavity and nasal cavity were lined with stratified parakeratinized squamous epithelial tissue and pseudo-stratified ciliate columnar epithelial tissue with caliciform cells, respectively. Between these epithelial tissues, the presence of connective tissue and bone tissue in the process of neoformation was evidenced in the premaxilla region. The nasal septum is in the central portion of the premaxilla and made up of hyaline cartilage and intra-membranous ossification, whereas ossification in the region of nasal conchae was endochondral. Tooth buds in the bell stage and delimited by the connective tissue of the nasopalatine region were evidenced. The developmental stage of these tooth buds was compatible with the age of the fetuses. The presence of cellular remnants of the dental lamina that formed the gubernacular cord and the trajectory of the incisive canal were also evidenced (Figure 2A).

The incisive canal in the formation stage was observed in all specimens as an opening delimited laterally by the periosteum and connecting the oral and nasal cavities. Near the nasal region, the incisive canal was either $\mathrm{Y}$ or $\mathrm{V}$ shaped (Figure 3 ). Blood vessels, glands, nerves, epithelial remains, remnants of the nasopalatine duct and a large quantity of fibers were found within this canal in all the fetuses.

The nasopalatine duct is an epithelial structure

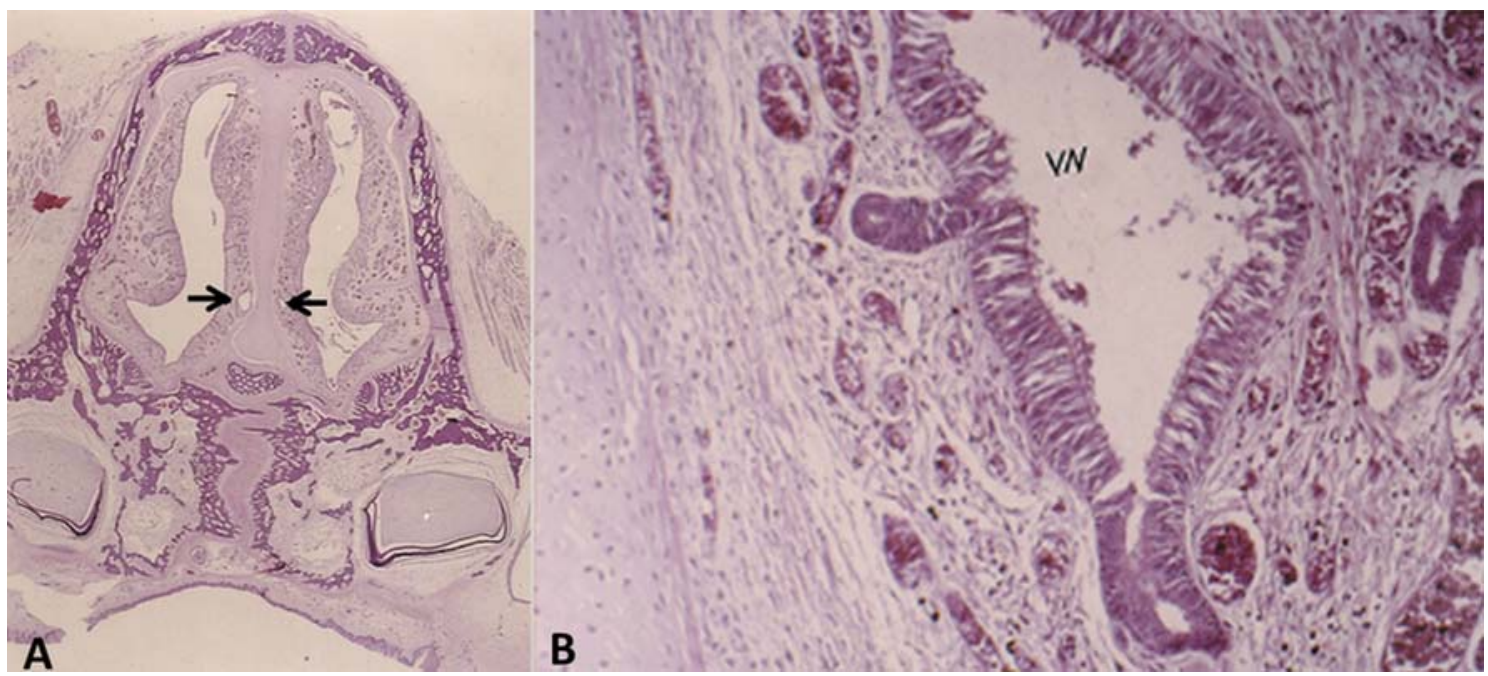

Figure 2- Oral and nasal cavity showing stratified parakeratinized squamous epithelial and pseudo-stratified ciliate columnar epithelial tissue. Presence of connective tissue and bone tissue in process of neoformation. The nasal septum in the central portion of the premaxilla and tooth buds in the bell stage. Bilateral vomeronasal organ (arrows) located alongside nasal septum (A); Vomeronasal organ and its constitution formed by tubular structure with pseudo-stratified ciliate epithelium; note associated blood vessels and nerves (B) [A: X1.25 magnification, hematoxylin \& eosin (HE); B: X3.2 magnification, HE) 


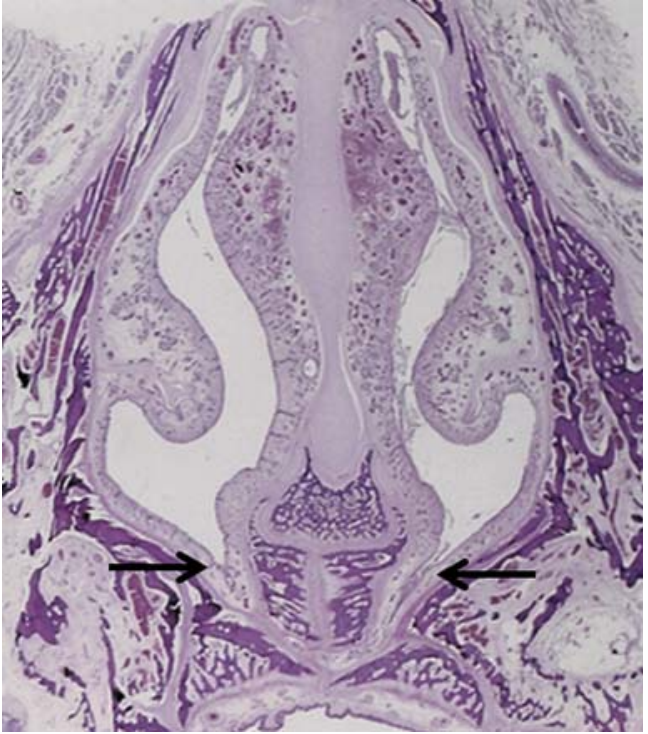

Figure 3- Y-shaped incisive canal and nasopalatine duct (arrows) opening into nasal cavity (X1.25 magnification, hematoxylin \& eosin) with a heterogeneous pattern. In one fetus, a bilateral nasopalatine duct was identified beginning at the floor of the nasal cavity. The duct was completely patent on one side and partially patent on the other (Figure 4). Structures similar to the nasopalatine duct were observed in all the other fetuses, located along the incisive canal. These structures had a larger diameter, similar to a cystic cavity (Figure 4). The mucous glands were found near the nasal mucosa, where their ducts opened. One patent nasopalatine duct was islet-shaped, with mucous glands opening in its interior.

The vomeronasal organ is a bilateral epithelialized structure located alongside the nasal septum above the incisive canal in all the fetuses. This organ is made up of a tubular structure with a pseudostratified ciliate epithelium. The ducts of mucous glands opened in its interior. Blood vessels and nerves are associated to this organ (Figure $2 \mathrm{~A}$ and $\mathrm{B}$ ).

The oral mucosa nearly always had a normal

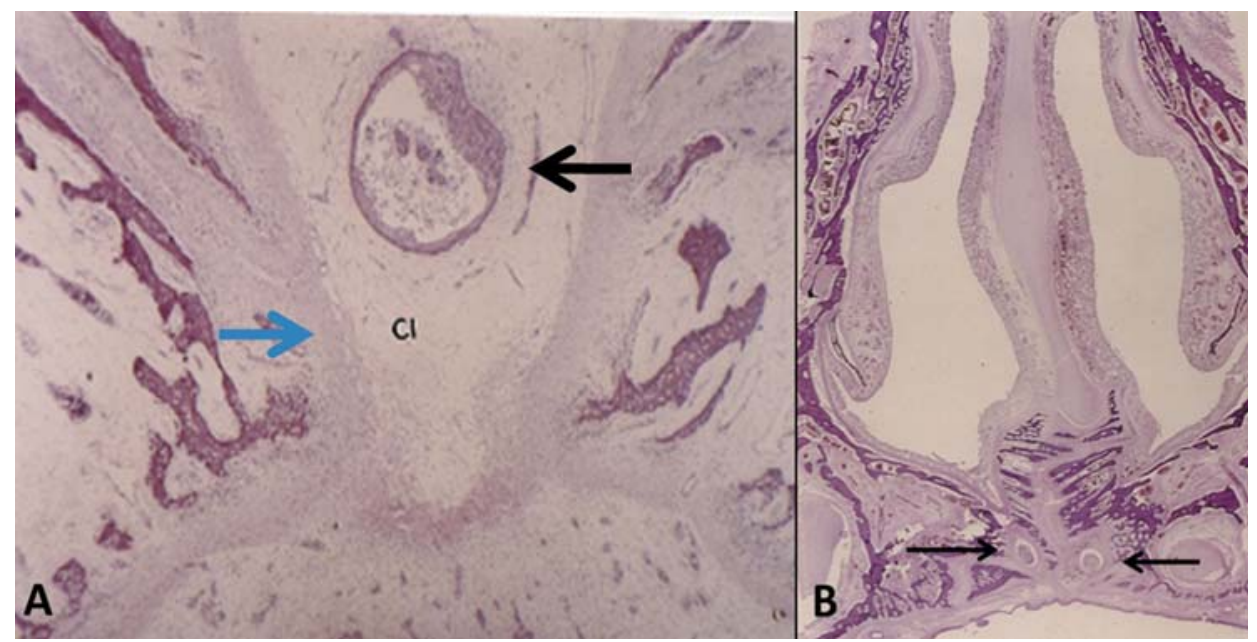

Figure 4- Persistent nasopalatine duct (black arrow) located in central portion of incisive canal in A (blue arrow). Bilateral persistent nasopalatine duct (black arrows) located in incisive canal in B [A: X3.2 magnification, hematoxylin \& eosin (HE); B: X1.25X magnification, HE]

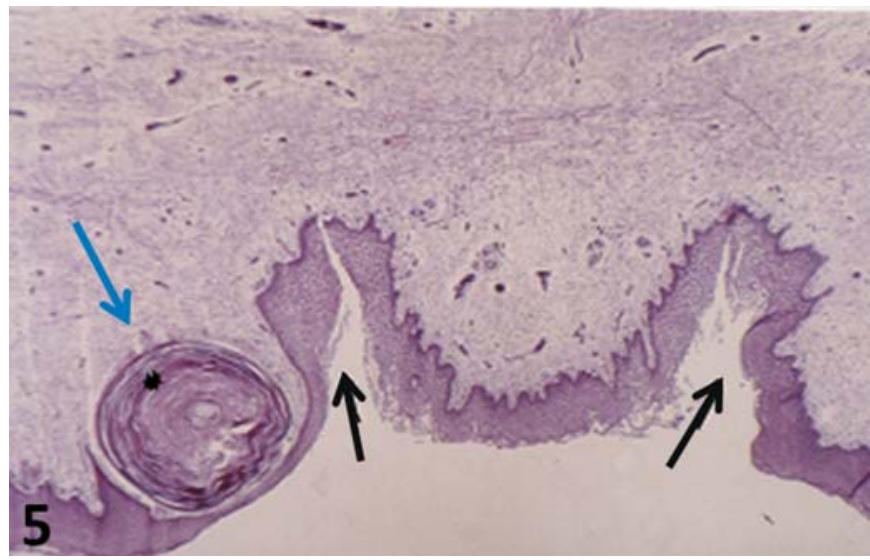

Figure 5- Horn cyst (blue arrow) in region of submucosa and bilateral nasopalatine duct entrance (black arrows) (X3.2 magnification, hematoxylin \& eosin) 
aspect and parakeratinized lining. In the presence of horn cysts in the submucosa, the lining epithelium of the oral mucosa had an atrophic pattern (Figure 5). Epithelial remnants, microcysts, blood vessels, nerves and glands were found in the submucosa. The conjunctive tissue of the submucosa exhibited a moderately to highly fibrous pattern.

\section{DISCUSSION}

In the present study, the age of the fetuses ranged from 8.00 to 9.25 weeks of life, which determined a homogeneous pattern in the morphological findings among the samples. The age was determined based on the femur size ${ }^{11}$. Another method described in the literature for determining the age is based on the size of the central incisors ${ }^{21}$. This method was not employed in the present study, since the teeth must be individualized for the measurement of the incisors using calipers.

Variation in shape and occurrence was observed in our fetuses with regard to the nasopalatine duct, which corroborates with clinical reports on the duct described in the literature ${ }^{4,9,13,23}$. This finding reveals an undefined behavior of development and persistence of the nasopalatine duct in human fetuses. Totally patent and partially patent nasopalatine ducts and their remnants in the form of islets or strings were observed. The presence of the nasopalatine duct or remnants of this duct was observed in the interior of the incisive canal both in the portion near the incisive papilla and near the nasal cavity. This suggests that the development of non-odontogenic cysts in the anterior region of the maxilla has its etiology in the cellular remains of the nasopalatine duct. The observation of epithelial remnants in the nasopalatine duct near the incisive papilla in human fetuses suggests the possibility that incisive papilla cysts originate from the embryological remains of the nasopalatine duct. This brings into question the denomination of a cyst in the nasopalatine region as a nasopalatine canal cyst, nasopalatine cyst or median palatine cyst ${ }^{8,10,17,24}$. The nomenclature of these cysts should be based on etiophatogenesis rather than anatomic location. Based on the results of the present study, the correct nomenclature would be a nasopalatine duct cyst, which corroborates with a number of studies found in the literature ${ }^{1,4,6,8,10,11,17,20-24}$.

The nasopalatine duct should not be confused with the incisive canal, since the duct is one of the structures present in the interior of the canal, as described in the present study. This divergence in conceptualization may be caused by the fact that the duct is sometimes obliterated in human adults ${ }^{16}$. Another divergence comes from the biological concept of the term duct. The authors of a recent study report two clinical cases of "oronasal fistula of the incisive papilla" and state that duct is a term used to define a tube formed by epithelial lines, characteristic of which is the conduction of fluids ${ }^{5}$. The authors stress that the correct term for the anatomic structure should be fistula rather than duct, since this structure may not have the function of conducting fluids, making it obliterated. However, this structure in the majority of mammals behaves as a duct and has the function of carrying pheromones from the oral cavity to the nasal cavity ${ }^{18,19}$. In humans, this structure is a holdover in the evolutional chain of the nasopalatine duct found in animals and it is suggested that it be classified as a nasopalatine duct in humans as well ${ }^{2}$.

In nearly all human fetuses analyzed in the present study, the vomeronasal organ was present bilaterally and was associated to the blood vessels and nerves. This suggests that this organ may be responsible for capturing external signals, even in humans, thereby placing into question whether it is truly a vestigial structure without function in humans. A recent study has demonstrated that the vomeronasal organ may not be part of the olfactory system, but rather an isolated structure ${ }^{15}$. Moreover, a comparative study using immunohistochemical processing of the vomeronasal organ in humans, other primates and mammals that use this organ for capturing pheromones suggest that the function of this organ is different in these mammals when compared to humans and other primates ${ }^{12}$.

The presence of incisive canals was found in all the fetuses of the present study and glands, nerves, remnants of the nasopalatine duct and a large quantity of fibers were found in the interior of this canal. The incisor canals were bilateral and near the nasal cavity in the shape of a $Y$ or $U$, as reported in previous studies $7,9,13,15,16,20,21,23$. Since the shape and histological characteristics of the incisive canal exhibited a homogeneous pattern among the fetuses, the possibility of lesions in this region originating from this anatomic structure can be discarded.

The oral mucosa, submucosa, ossification and dental structures did not exhibit significant alterations from one fetus to another. However, an atrophic pattern was observed in the oral mucosa in one fetus, in which a horn cyst was found in the submucosa region. The persistence of this lesion may lead to the development of the cyst in the newborn ${ }^{3,14}$. This pathology is classified as Bohn's nodule, Epstein's pearls or dental lamina cyst. These conditions are considered synonyms due to their clinical-pathological characteristics and differ only with regard to the site of occurrence ${ }^{3}$. Such alterations appear within the first year of life and are resolved spontaneously without the need for intervention. 


\section{CONCLUSION}

The present study allows the inference that the incisive canal, nasopalatine duct and vomeronasal organ are distinct anatomic structures. Moreover, the development of nasopalatine duct cysts may be related to the persistent, totally patent or partially patent forms of the nasopalatine duct and its cell remnants found in the interior of the incisive canal.

\section{REFERENCES}

1- Aka PS, Canturk N, Dagalp R, Yagan M. Age determination from central incisors of fetuses and infants. Forensic Sci Int. 2009;184:15-20.

2- Bellairs AD. Observations on the incisive canaliculi and nasopalatine ducts. Br Dent J. 1951;91:281-91.

3- Cambiaghi S, Gelmetti C. Bohn's nodules. Int J Dermatol. 2005;44:753-4.

4- Catros S, De Gabory L, Stoll D, Deminière C, Fricain JC. Use of gutta percha cores in CT scan imaging for patent nasopalatine duct. Int J Oral Maxillofac Surg. 2008;37:1065-6.

5- Edwards PC, Kanjirath PP, Norton NS, McVaney T, Scanlon C, Saini T. Developmental oronasal fistula of the incisive papilla. Gen Dent. 2010;58:62-7.

6- Escoda Francolí J, Almendros Marqués N, Berini Aytés L, Gay Escoda C. Nasopalatine duct cyst: report of 22 cases and review of the literature. Med Oral Patol Oral Cir Bucal. 2008; 13:E438-43. 7- Foltán R, Sedý J. Behavioral changes of patients after orthognathic surgery develop on the basis of the loss of the vomeronasal organ: a hypothesis. Head Face Med. 2009;5:5.

8- Hadi U, Younes A, Ghosseini S, Tawil A. Median palatine cyst: an unusual presentation of a rare entity. $\mathrm{Br}$ ] Oral Maxillofac Surg. 2001;39:278-81.

9- Jacob S, Zelano B, Gungor A, Abbott D, Naclerio R, McClintock MK. Location and gross morphology of the nasopalatine duct in human adults. Arch Otolaryngol Head Neck Surg. 2000;126:741-8. 10- Karaçal N, Ambarcoğlu O, Kutlu N. Median palatine cyst: report of an unusual entity. Plast Reconstr Surg. 2005;115:1213-4.
11- Kelemen E, Jánossa M, Calvo W, Fliedner TM. Developmental age estimated by bone-length measurement in human fetuses. Anat Rec. 1984;209:547-52.

12- Kinzinger JH, Johnson EW, Bhatnagar KP, Bonar $\mathrm{CJ}$, Burrows AM, Mooney MP, et al. Comparative study of lectin reactivity in the vomeronasal organ of human and nonhuman primates. Anat Rec A Discov Mol Cell Evol Biol. 2005;284:550-60.

13- Knecht M, Kittner T, Beleites T, Hüttenbrink KB, Hummel T, Witt M. Morphological and radiologic evaluation of the human nasopalatine duct. Ann Otol Rhinol Laryngol. 2005;114:229-32. 14- Lewis DM. Bohn's nodules, Epstein's pearls, and gingival cysts of the newborn: a new etiology and classification. J Okla Dent Assoc. 2010;101:32-3.

15- Martinez-Marcos A. On the organization of olfactory and vomeronasal cortices. Prog Neurobiol. 2009;87:21-30.

16- Radlanski RJ, Emmerich S, Renz H. Prenatal morphogenesis of the human incisive canal. Anat Embryol (Berl). 2004;208:265-71. 17- Righini CA, Boubagra K, Bettega G, Verougstreate G, Reyt E. Nasopalatine canal cyst: 4 cases and a review of the literature. Ann Otolaryngol Chir Cervicofac. 2004;121:115-9.

18- Schneider NY, Fletcher TP, Shaw G, Renfree MB. The vomeronasal organ of the tammar wallaby. J Anat. 2008;213:93105.

19- Shimp KL, Bhatnagar KP, Bonar CJ, Smith TD. Ontogeny of the nasopalatine duct in primates. Anat Rec A Discov Mol Cell Evol Biol. 2003;274:862-9.

20- Song WC, Jo DI, Lee JY, Kim JN, Hur MS, Hu KS, et al. Microanatomy of the incisive canal using three-dimensional reconstruction of microCT images: an ex vivo study.Oral Surg Oral Med Oral Pathol Oral Radiol Endod. 2009;108:583-90.

21- Tanaka S, Iida S, Murakami S, Kishino M, Yamada C, Okura $M$. Extensive nasopalatine duct cyst causing nasolabial protrusion. Oral Surg Oral Med Oral Pathol Oral Radiol Endod. 2008;106:e4650.

22- Tsuneki M, Maruyama S, Yamazaki M, Abé T, Adeola H, Cheng J, et al. Inflammatory histopathogenesis of nasopalatine duct cyst: a clinicopathological study of 41 cases. Oral Dis. 2013;19:415-24. 23- Valstar $\mathrm{MH}$, van den Akker HP. Patent nasopalatine duct: a diagnostic pitfall. Br J Oral Maxillofac Surg. 2008;46:304-5.

24- Velasquez-Smith MT, Mason C, Coonar H, Bennett J. A nasopalatine cyst in an 8-year-old child. Int J Paediatr Dent. 1999;9:123-7. 